\title{
COLORS, AGES, AND METALLICITIES OF GALAXIES IN SIX NEARBY GALAXY CLUSTERS
}

\author{
Jong Chul Lee, Myung Gyoon Lee, and Taehyun Kim \\ Astronomy Program, Department of Physics and Astronomy, Seoul National University, Seoul 151-742, Korea \\ E-mail: jclee@astro.snu.ac.kr,mglee@astrog.snu.ac.kr,8 thkim@astro.snu.ac.kr
}

(Received July 15, 2008; Accepted October 14, 2008)

\begin{abstract}
We present an optical-infrared photometric study of galaxies in six nearby clusters of galaxies at $\mathrm{z}=0.041 \sim 0.098$ (A1436, A1773, A1809, A2048, A2142, and A2152). Using BVI photometry obtained at the Bohyunsan Optical Astronomical Observatory and $J H K_{S}$ photometry extracted from the 2-Micron All-Sky Survey catalog, we investigate the colors of galaxies in the clusters. Using the $(B-V)$ versus $\left(I-K_{S}\right)$ color-color diagrams in comparison with the simple stellar population model, we estimate the ages and metallicities of bright early-type member galaxies. Early-type galaxies in each cluster show the color-magnitude relation. Ages and metallicities of early-type members show little dependence on their velocity dispersions. Mean ages of early-types in the clusters range from 3 Gyr to 20 Gyr, showing a large dispersion, and mean metallicities range from $Z=0.03$ to 0.05 above the solar value, showing a negligible dispersion.
\end{abstract}

Key words : galaxy clusters : individual (A1436, A1773, A1809, A2048, A2142, and A2152) galaxy : photometry — galaxy : color, age, and metallictiy — galaxy : formation and evolution

\section{INTRODUCTION}

One of the well-known observational properties of elliptical galaxies in nearby galaxy clusters is that they follow a tight color-magnitude relation (CMR): more luminous ellipticals tend to be redder (Sandage \& Visvanathan 1978). Nowadays the CMR is established in distant cluster up to $\mathrm{z} \sim 1$ (Holden et al. 2004), and is also found in high redshift proto-clusters even at $\mathrm{z} \sim 3$ (Kodama et al. 2007). One of the recent key issues on CMR is whether the CMR shows any dependence on environments (Hogg et al. 2004; Smith et al. 2006).

The CMR for nearby galaxy clusters shows small scatter in general and the intercept of the CMR shows modest color evolution. The small scatter in the CMR is often interpreted as evidence that galaxies were formed in a short period of time (Terlevich, Caldwell, \& Bower 2001) and the intercept evolution is consistent with the passively evolving stellar population formed at high redshift (Stanford, Eisenhardt, \& Dickinson 1998). These seem to support the monolithic collapse model for elliptical galaxy formation (Bower, Lucey, \& Ellis 1992), but Kauffmann \& Charlot (1998) argued that the CMR can be explained also in the context of hierarchical merging model. More details about the models of elliptical galaxy formation are summarized in Lee (2003).

It is generally accepted that the CMR is mainly driven by age and metallicity effects. The CMR may be attributed to metallicity sequence in the sense that

\footnotetext{
Corresponding Author: M. G. Lee
}

more massive galaxies are more metal-rich, because they can retain gas for longer than less massive galaxies (Kodama et al. 1998; Gallazzi et al. 2006). On the other hand, it is also possible to explain part of the CMR as an increase in the mean stellar age with luminosity (Ferreras, Charlot, \& Silk 1999).

To understand the origin of the CMR and how elliptical galaxies formed, we need to estimate the ages and metallicities of elliptical galaxies. The integrated color of stellar populations becomes generally red as they get old, thus stellar ages of galaxies can be estimated from the color information of the galaxies. However, more metal rich stars can also make their host galaxy redder. Therefore we need to know the effects of age and metallicity, respectively, but it is not easy to do so. The degeneracy between age and metallicity is widely known as the $3 / 2$ rule (Worthey 1994): if two populations have $\Delta \log (A g e) / \Delta \log (Z) \sim-3 / 2$, then they will look alike in nearly every broad-band color and line index.

One of the ways to break this degeneracy is to use the combination of optical and near-infrared (NIR) photometry (Aaronson 1978). Optical-NIR color of an old stellar population is determined mainly by the temperature of the red giant branch stars so that it is more sensitive to metallicity than to age, while its optical color depends upon both age and metallicity. Therefore we can distinguish between age and metallicity effects using the combination of optical and NIR color. Smail et al. (2001) applied this method to Abell 2218 at $z=0.176$. They obtained $(B-I),(V-I)$, and 
$\left(I-K_{S}\right)$ colors of galaxies in A2218, and estimated the ages and metallicities of the galaxies by comparing these colors with the simple stellar population model. They concluded that luminous early-type galaxies trace a sequence of varying metallicity at a single age, while faint early-type galaxies show a large spread in their ages and metallicities.

With the advent of several wide-field surveys such as Sloan Digital Sky Survey (SDSS; York et al. 2000) and National Optical Astronomy Observatory Fundamental Plane Survey (Smith et al. 2004), it became possible to estimate spectroscopically the ages and metallicities for a large number of galaxies. Using these survey data, several studies found that more massive galaxies are older and more metal-rich than less massive galaxies (Bernardi et al. 2005; Gallazzi et al. 2005, 2006; Nelan et al. 2005; Jimenez et al. 2007). However most of these studies covered a large sample of galaxies in the surveys without separating cluster galaxies, and there are few systematic studies focusing on ages and metallicities of cluster galaxies.

We have been carrying out a project to study the ages and metallicities of galaxies in nearby clusters photometrically and spectroscopically. Spectroscopic method and photometric method have both pros and cons. It is considered that spectroscopic measurement of age and metallcity is more reliable than photometric measurement. However it is hard to get high quality spectra for all cluster members, and the galaxy properties derived from the spectra are confined to the central region of a galaxy, due to the small size of the apertures. On the other hand, photometric method can be applied to all bright member galaxies and can cover the large fraction of a galaxy. In this study we present results for six nearby Abell clusters based on photometric method only. We display optical and NIR photometry for galaxies in these clusters. We obtained optical-NIR colors of the galaxies by combining the optical observational data and the NIR data in the 2 Micron All-Sky Survey (2MASS; Kleinmann et al. 1994) Extended Source Catalog (XSC; Jarrett et al. 2000). We estimated the ages and metallicities of the galaxies by comparing the optical-NIR colors with the simple stellar population model. This paper is organized as follows. Section II describes observation and data reduction. In Section III, we classify the galaxies and select the cluster members. Then we present the color-magnitude diagrams and color-color diagrams, and estimate the ages and metallicities of the galaxies. Primary results are discussed and summarized in Section IV and V, respectively. Throughout this paper, we assumed a cosmology with $\Omega_{M}=0.3, \Omega_{\Lambda}=0.7$, and $H_{0}=72 \mathrm{~km} \mathrm{~s}^{-1} \mathrm{Mpc}^{-1}$.

\section{OBSERVATION AND DATA REDUCTION}

We selected six nearby galaxy clusters in the Abell catalog (Abell, Corwin, \& Olowin 1989). Selection criteria for the targets are: (a) they should be nearby at $z<0.1$ so that we can secure precise photome- try of cluster galaxies using the $1.8 \mathrm{~m}$ telescope; (b) they should be rich and contain several bright elliptical galaxies so that we can do statistical analysis of galaxies; and (c) some of them may have interesting properties (e.g., high X-ray luminosity, merging features, or cD galaxies). The basic information of the cluster sample is summarized in Table 1. Among these, A2142 is one of the well-known merging galaxy clusters (Markevitch et al. 2000). This cluster is X-ray bright and has two giant elliptical galaxies near the center. In the region of A2152, there is a background cluster at $\mathrm{z} \sim$ 0.13 , whose core is closely aligned with that of A2152 (Blakeslee et al. 2001).

We observed the target clusters using the SITe $2 \mathrm{~K}$ CCD camera and $B V I$ filters (Johnson-Cousins filter system; Bessell 1990) at the Bohyunsan Optical Astronomical Observatory (BOAO) $1.8 \mathrm{~m}$ telescope in Korea on May $2-4,2005$. The size of the field of view is $11^{\prime} .7$ $\times 11^{\prime} .7$, and the pixel scale is $0^{\prime \prime} .3438$ pixel $^{-1}$. Seeing ranged around $2^{\prime \prime}$, so we used $2 \times 2$ binning mode during the observation. We observed two to four fields around the center of each cluster. Table 2 lists the observation log, and Figure 1 displays gray-scale maps of the $V$ band images for the observed fields.

Raw images were bias-subtracted and flat-fielded using the IRAF* tasks. Twilight sky flat images obtained on the same night as the object images were used for flat-fielding. We combined the individual images using median values to make a master image for each field. We used the Source Extractor (SExtractor) package (Bertin \& Arnouts 1996) to detect the sources and obtain their instrumental magnitudes in the master images. We used $5 \sigma$ detection threshold and a minimum pixel number of 4 for source detection. To obtain the color of a galaxy with the same shape of apertures, we ran the SExtractor in the dual-image mode, taking the $V$ band image as a reference frame. Among the output parameters from SExtractor, we used MAG_AUTO (Kron-like elliptical aperture magnitude) for a galaxy magnitude. We selected the galaxies in the output list using the CLASS_STAR parameter (stellarity) calculated by SExtractor. The stellarity values run from 0 for extended sources to 1 for point sources. Bertin \& Arnouts (1996) recommended 0.5 as the star-galaxy separation value, which was adopted in this study.

The instrumental magnitudes were transformed into the standard system using the standard stars (Landolt 1992) observed on the same night. The root mean squares of the transformations are on average 0.032 for $B, 0.024$ for $V$, and 0.034 for $I$. Then these magnitudes were corrected for Galactic extinction and the K-correction. We used the value of foreground Galactic extinction for each object provided by Schlegel, Finkbeiner, \& Davis (1998). Since the ex-

* IRAF is distributed by the National Optical Astronomy Observatories, which are operated by the Association of Universities for Research in Astronomy, Inc., under cooperative agreement with the National Science Foundation. 

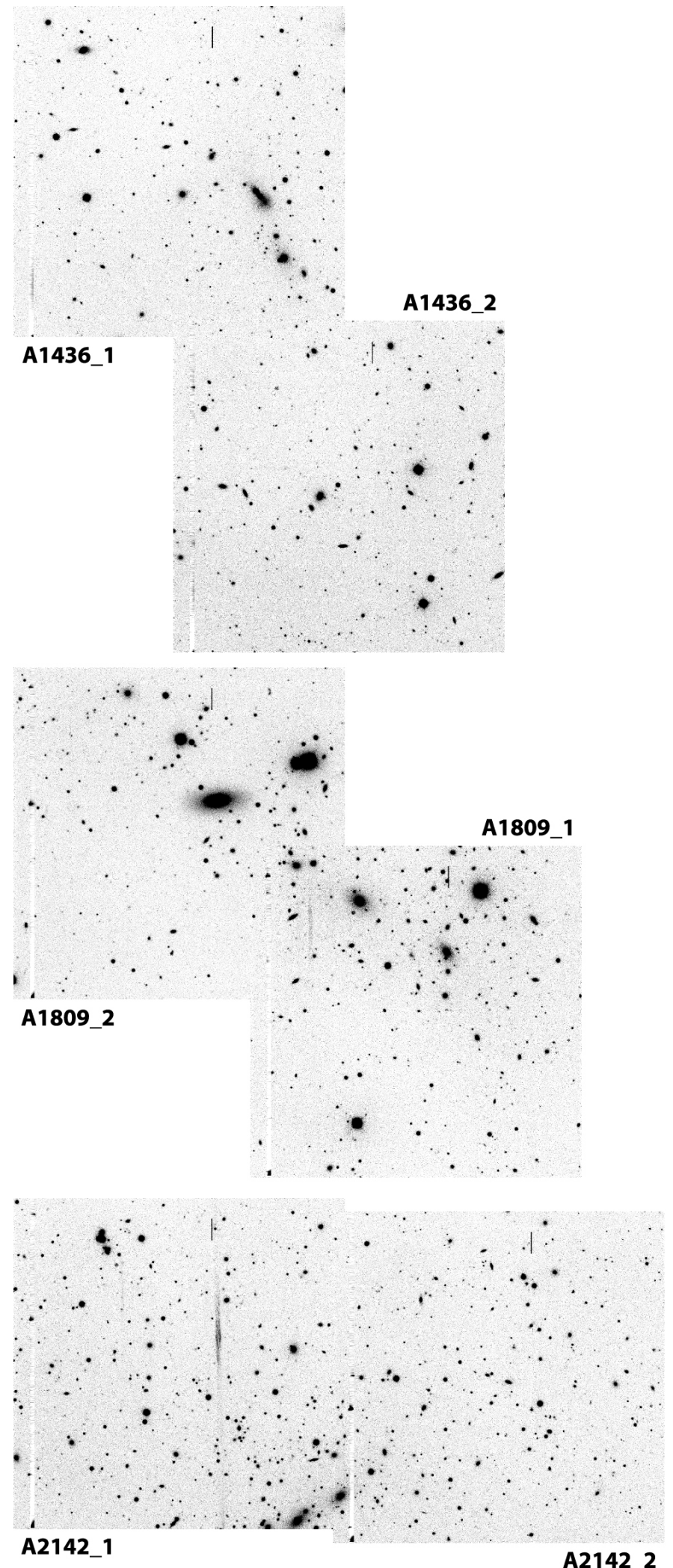

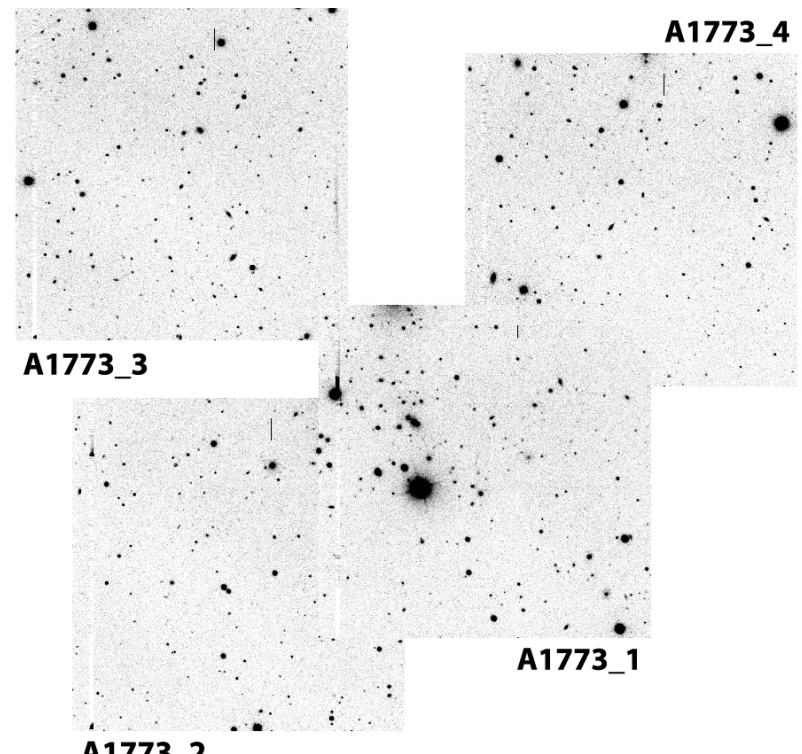

A1773_2
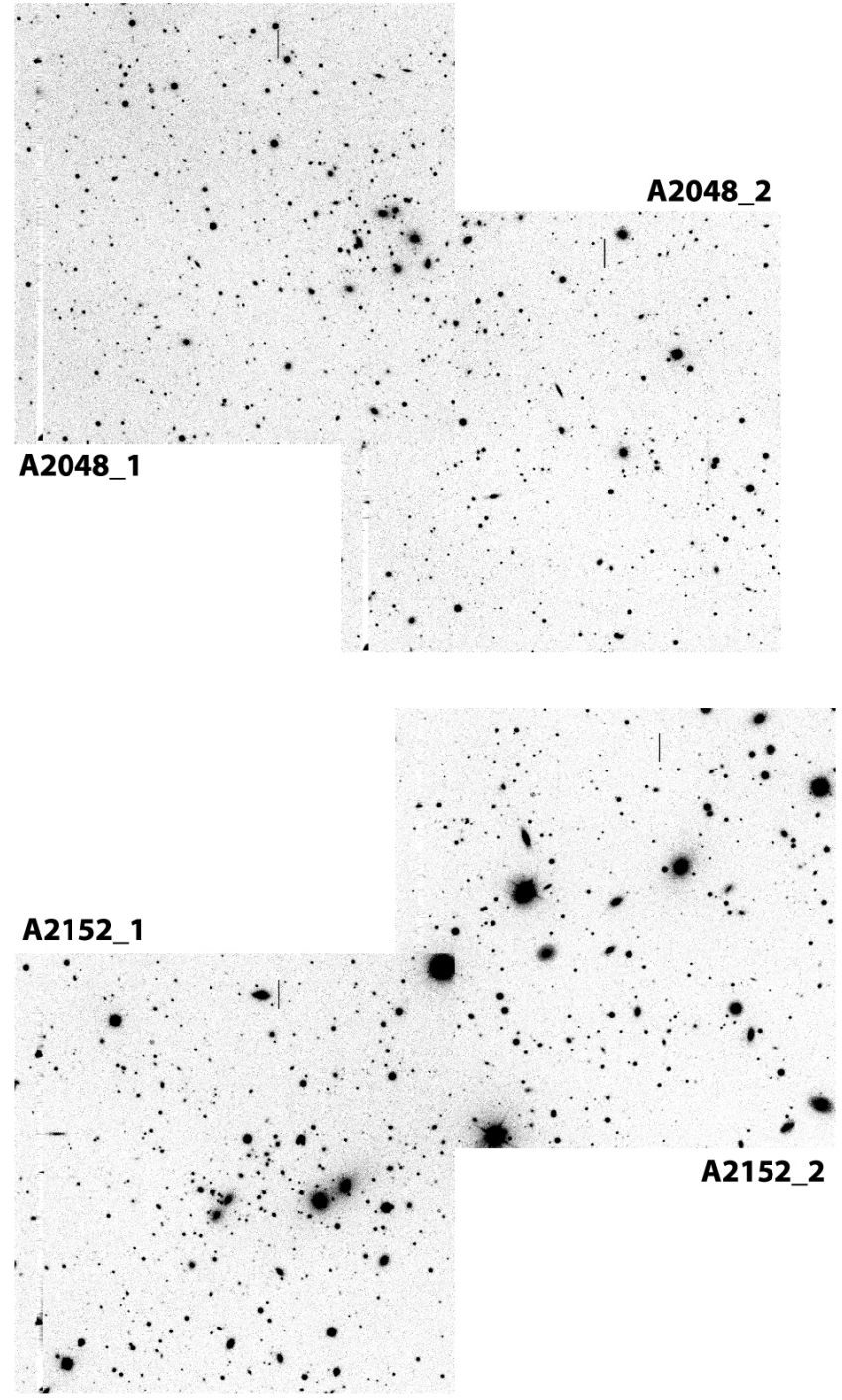

Fig. 1 - Gray-scale maps of the $V$ band images of six nearby galaxy clusters. The size of each field is $11^{\prime} .7 \times 11^{\prime} .7$, and the center position of each field is given in Table 2. North is up and east is to the left. 
Table 1. Basic Information of the Target Clusters

\begin{tabular}{|c|c|c|c|c|c|c|c|c|c|}
\hline Cluster & $\alpha, \delta$ & $\mathrm{J} 2000)^{\mathrm{a}}$ & $m_{10}{ }^{\mathrm{a}}$ & $\mathrm{R}^{\mathrm{a}}$ & $\mathrm{BM}^{\mathrm{b}}$ & $\mathrm{E}(B-V)^{\mathrm{c}}$ & $\mathrm{z}^{\mathrm{d}}$ & $\sigma_{r}[\mathrm{~km} / \mathrm{s}]^{\mathrm{d}}$ & $\mathrm{N}^{\mathrm{d}}$ \\
\hline A1436 & $12^{h} 00^{m} 28^{s}$ & $+56^{\circ} 15^{\prime} 18^{\prime \prime}$ & 15.4 & 1 & III & 0.018 & 0.0644 & 501 & 28 \\
\hline A1773 & $13^{h} 42^{m} 08^{s}$ & $+02^{\circ} 14^{\prime} 54^{\prime \prime}$ & 15.6 & 1 & III & 0.029 & 0.0769 & 845 & 41 \\
\hline A1809 & $13^{h} 53^{m} 19^{s}$ & $+05^{\circ} 09^{\prime} 18^{\prime \prime}$ & 15.8 & 1 & II: & 0.026 & 0.0795 & 780 & 50 \\
\hline A 2048 & $15^{h} 15^{m} 18^{s}$ & $+04^{\circ} 22^{\prime} 54^{\prime \prime}$ & 16.0 & 1 & III & 0.048 & 0.0977 & 864 & 65 \\
\hline A2142 & $15^{h} 58^{m} 16^{s}$ & $+27^{\circ} 13^{\prime} 30^{\prime \prime}$ & 16.0 & 2 & II & 0.044 & 0.0897 & 1000 & 190 \\
\hline A 2152 & $16^{h} 05^{m} 22^{s}$ & $+16^{\circ} 26^{\prime} 54^{\prime \prime}$ & 13.8 & 1 & III & 0.040 & 0.0410 & 1338 & 62 \\
\hline
\end{tabular}

a Cluster position, red magnitude for the 10th brightest member, and richness class (Abell, Corwin, \& Olowin 1989).

${ }^{b}$ Classification in the Bautz-Morgan system (Bautz \& Morgan 1970).

${ }^{c}$ Amount of foreground Galactic extinction (Schlegel, Finkbeiner, \& Davis 1998).

$d$ Adopted redshift, radial velocity dispersion, and the number of galaxies used to determine the redshift and velocity dispersion (The values of A2152 are taken from Struble \& Rood 1999 and the others are from Yoon et al. 2008.).

Table 2. Observation Log

\begin{tabular}{|c|c|c|c|c|c|c|c|c|c|c|c|}
\hline \multirow[t]{2}{*}{ Field ID } & \multirow{2}{*}{$\begin{array}{l}\text { Obs. Date } \\
\text { (UT) }\end{array}$} & \multicolumn{3}{|c|}{ Coordinates (J2000) } & \multirow{2}{*}{$\begin{array}{c}\text { Airmass } \\
(\mathrm{X})\end{array}$} & \multicolumn{2}{|c|}{ Exposure time } & \multirow{2}{*}{$\frac{\text { [sec] }}{I}$} & \multicolumn{3}{|c|}{ Seeing ["] } \\
\hline & & & R.A. & Dec. & & $B$ & $V$ & & $B$ & $V$ & $I$ \\
\hline A1436_1 & May. 2 & $12^{h}$ & $00^{m} 59^{s}$ & $+56^{\circ} 15^{\prime} 49^{\prime \prime}$ & 1.07 & $110 \times 5$ & $80 \times 3$ & $35 \times 3$ & 2.0 & 2.0 & 2.0 \\
\hline A1436_2 & May. 2 & $12^{h}$ & $00^{m} 16^{s}$ & $+56^{\circ} 04^{\prime} 58^{\prime \prime}$ & 1.08 & $120 \times 5$ & $100 \times 3$ & $45 \times 3$ & 1.9 & 1.8 & 1.8 \\
\hline A1773_1 & May. 3 & $13^{h}$ & $42^{m} 16^{s}$ & $+02^{\circ} 10^{\prime} 14^{\prime \prime}$ & 1.33 & $70 \times 9$ & $45 \times 7$ & $25 \times 5$ & 2.7 & 2.8 & 2.1 \\
\hline A1773_2 & May. 3 & $13^{h}$ & $42^{m} 51^{s}$ & $+02^{\circ} 06^{\prime} 53^{\prime \prime}$ & 1.24 & $140 \times 5$ & $110 \times 3$ & $30 \times 5$ & 2.3 & 2.3 & 2.1 \\
\hline A1773_3 & May. 3 & $13^{h}$ & $43^{m} 00^{s}$ & $+02^{\circ} 20^{\prime} 30^{\prime \prime}$ & 1.21 & $150 \times 5$ & $130 \times 3$ & $50 \times 3$ & 2.4 & 2.3 & 1.8 \\
\hline A1773_4 & May. 3 & $13^{h}$ & $41^{m} 59^{s}$ & $+02^{\circ} 19^{\prime} 02^{\prime \prime}$ & 1.22 & $100 \times 7$ & $70 \times 5$ & $30 \times 5$ & 2.9 & 2.6 & 2.6 \\
\hline A1809_1 & May. 2 & $13^{h}$ & $53^{m} 15^{s}$ & $+05^{\circ} 03^{\prime} 29^{\prime \prime}$ & 1.17 & $150 \times 5$ & $110 \times 3$ & $45 \times 3$ & 2.2 & 2.3 & 1.8 \\
\hline A1809_2 & May. 2 & $13^{h}$ & $53^{m} 49^{s}$ & $+05^{\circ} 09^{\prime} 35^{\prime \prime}$ & 1.17 & $130 \times 5$ & $110 \times 3$ & $40 \times 3$ & 2.7 & 2.6 & 2.1 \\
\hline A2048_1 & May. 3 & $15^{h}$ & $15^{m} 50^{s}$ & $+04^{\circ} 22^{\prime} 09^{\prime \prime}$ & 1.19 & $140 \times 5$ & $120 \times 3$ & $40 \times 3$ & 2.3 & 2.2 & 2.1 \\
\hline A2048_2 & May. 3 & $15^{h}$ & $15^{m} 16^{s}$ & $+04^{\circ} 16^{\prime} 43^{\prime \prime}$ & 1.24 & $140 \times 5$ & $120 \times 3$ & $45 \times 3$ & 2.0 & 1.9 & 1.8 \\
\hline A2142_1 & May. 2 & $15^{h}$ & $58^{m} 54^{s}$ & $+27^{\circ} 18^{\prime} 11^{\prime \prime}$ & 1.05 & $150 \times 5$ & $130 \times 3$ & $50 \times 3$ & 2.8 & 2.5 & 2.3 \\
\hline A2142_2 & May. 2 & $15^{h}$ & $58^{m} 04^{s}$ & $+27^{\circ} 17^{\prime} 49^{\prime \prime}$ & 1.14 & $140 \times 5$ & $120 \times 3$ & $45 \times 3$ & 2.1 & 2.0 & 2.1 \\
\hline A2152_1 & May. 2 & $16^{h}$ & $05^{m} 54^{s}$ & $+16^{\circ} 25^{\prime} 54^{\prime \prime}$ & 1.07 & $110 \times 5$ & $90 \times 3$ & $35 \times 3$ & 2.6 & 2.4 & 2.2 \\
\hline A2152_2 & May. 2 & $16^{h}$ & $05^{m} 13^{s}$ & $+16^{\circ} 32^{\prime} 20^{\prime \prime}$ & 1.06 & $140 \times 5$ & $110 \times 3$ & $40 \times 3$ & 2.4 & 2.3 & 2.0 \\
\hline
\end{tabular}

tinction data in $K_{S}$ band was not available, we used the value for $K$ band. The difference between the effective wavelengths of $K$ and $K_{S}$ is small enough that the resulting difference in extinction is negligible. The K-correction was performed with kcorrect.v3_2 code (Blanton et al. 2003). We used spectroscopic redshifts from the galaxy catalog of the SDSS 6th Data Release (Adelman-McCarthy et al. 2008) for the K-correction and for the calculation of the luminosity distance to a galaxy, when its redshift is available. If there is no redshift information for a galaxy, we assume the redshift of the galaxy is the same as that of its host cluster. Finally the apparent magnitudes were converted into absolute magnitudes using the luminosity distances.

We used the 2MASS XSC released in March 2003 for the NIR photometric study. 2MASS project performed all-sky observation in 3 bands $(J: 1.25 \mu \mathrm{m}$, $H: 1.65 \mu \mathrm{m}$, and $\left.K_{S}: 2.17 \mu \mathrm{m}\right)$ using $1.3 \mathrm{~m}$ telescopes at Mt. Hopkins and Cerro Tololo. In this study, we used the fiducial Kron elliptical aperture magnitude for a galaxy magnitude (Kron 1980), for which the aperture was defined in $K_{S}$ band.

\section{RESULTS}

\section{(a) Galaxy Classification and Cluster Member Selection}

We classified the morphology of bright and large galaxies, which are in both the BOAO observation and the 2MASS extended source catalog. The galaxies not found in the 2MASS catalog were too faint or too small to determine their morphological types, so we could not classify them. The 2MASS magnitude limit is $K_{S}=$ $14.5 \mathrm{mag}$, and corresponds to $17.8 \mathrm{mag}$ in $V$ band for red sequence galaxies with $\left(V-K_{S}\right) \sim 3.3$ mag. Among the galaxies brighter than $\mathrm{V}=17.8 \mathrm{mag}$, the fractions 


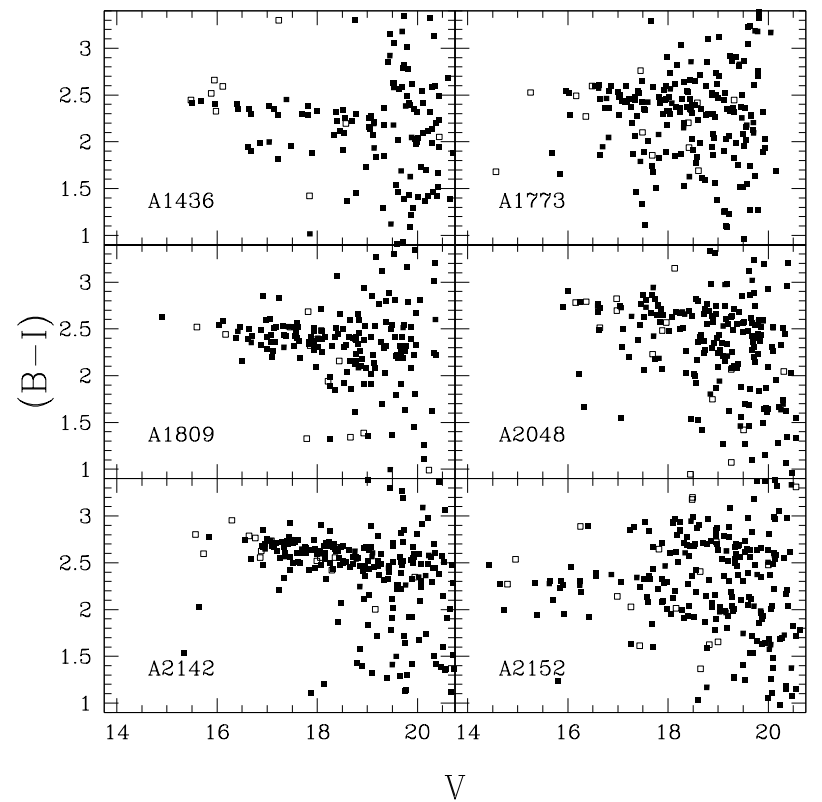

Fig. 2-V - $(B-I)$ color-magnitude diagrams of the detected galaxies in six clusters. Filled symbols represent the isolated galaxies with SExtractor flag=0 (i.e., the sources without any neighbor within the aperture used).

of unclassified galaxies in target clusters are $8 \sim 21 \%$. Two of the authors (J. C. Lee \& T. Kim) classified the bright and large galaxies into early-types (ellipticals and S0s) and late-types (spirals and irregulars) independently via visual inspection of the combined CCD images. A comparison of the two results showed a good agreement with an agreement rate of $\sim 92 \%$. We classified together again the galaxies for which the classification results were different in the first try until both agreed. In addition, we smoothed the images of the nearest cluster A2152 using Gaussian convolution so that seeing-to-physical scale would be the same for the farthest cluster A2048, and checked the morphological types of A2152 galaxies on the images. Among the twenty one member galaxies, only one type was changed. Therefore we expect that the effect of scale variation on the classification is about 5 percent within our redshift coverage. The fractions of early-types in most clusters are $50 \sim 62 \%$. In A2142, the fraction is exceptionally large $(\sim 79 \%)$. It is noted that we observed the smaller core region of this cluster compared with other clusters.

We selected the member galaxies of a cluster using the radial velocities of galaxies. We regarded a galaxy as a member of the cluster if its radial velocity with respect to the radial velocity of the cluster is within twice the radial velocity dispersion of the cluster. The galaxies whose spectroscopic redshifts are not available were considered as uncertain members. We did not use any spatial boundary conditions for member selection

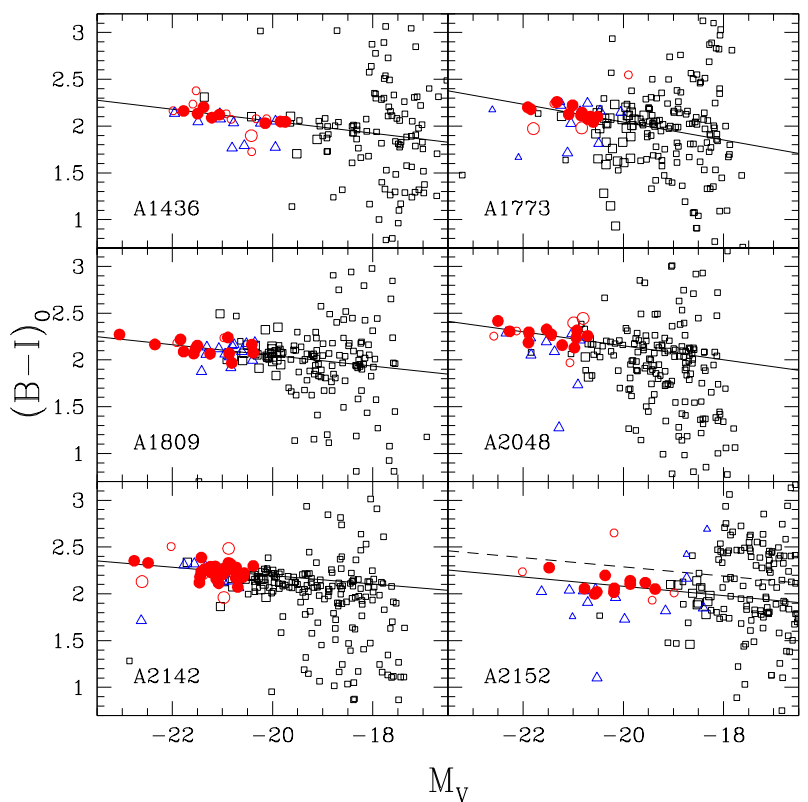

Fig. $\quad 3-M_{V}-(B-I)_{0}$ color-magnitude diagrams of the galaxies in six clusters (circles : early-type galaxies, squares : unclassified galaxies, and triangles : latetype galaxies). Large symbols and small symbols represent confirmed members and uncertain members, respectively. Large filled circles represent the data used for a linear fit of the CMR that is plotted by the straight line, while large open circles represent the data not used for fitting. The dashed line in the color-magnitude diagram of A2152 indicates a boundary for separating galaxies in A2152 from background cluster galaxies.

because the observed fields (radius : $\sim 12$ arcmin) are smaller than the size of clusters. According to Yoon et al. (2008), the virial radii of A1436, A1773, A1809, and A2048 are 14 16 arcmin, and that of A2142 is 29 arcmin. Although the virial radius information of A2152 is not accessible, we expect that the observed fields of A2152 are also smaller than the cluster size in the sense that the typical radius of a galaxy cluster is $\sim 2.0 \mathrm{Mpc}$ (Cox 2000), corresponding to an angular size of 41 arcmin at $z=0.041$. If the galaxy properties change as a function of cluster-centric distance, our analysis with different covering fractions could be biased. When we used the galaxies within a consistent radius $(40 \%$ of virial radius) among the clusters, luminosity-weighted mean colors of early-type galaxies were not significantly changed. On the other hand, CMR parameters became more uncertain due to decreasing the number of fitted galaxies, and the early-type fractions increased to $70 \sim 80 \%$. Thus we concluded that our main results are not affected by a radial cut, and used all galaxies in the observed fields. 
Table 3. Linear Fit Parameters for the Color-Magnitude Relation

\begin{tabular}{cccccccc}
\hline \hline & Color & A1436 & A1773 & A1809 & A2048 & A2142 & A2152 \\
\hline $\mathrm{N}^{\mathrm{a}}$ & & $10(9)$ & $14(12)$ & $13(13)$ & $13(11)$ & $31(28)$ & $11(11)$ \\
\hline Slope & $(B-I)$ & $-0.064 \pm 0.024$ & $-0.096 \pm 0.058$ & $-0.057 \pm 0.022$ & $-0.075 \pm 0.049$ & $-0.045 \pm 0.021$ & $-0.049 \pm 0.029$ \\
& $(V-I)$ & $-0.041 \pm 0.019$ & $-0.014 \pm 0.056$ & $-0.042 \pm 0.017$ & $-0.058 \pm 0.046$ & $-0.035 \pm 0.016$ & $-0.023 \pm 0.022$ \\
& $(B-V)$ & $-0.023 \pm 0.020$ & $-0.082 \pm 0.058$ & $-0.015 \pm 0.018$ & $-0.018 \pm 0.049$ & $-0.010 \pm 0.018$ & $-0.027 \pm 0.024$ \\
Intercept & $(B-I)$ & $2.05 \pm 0.49$ & $2.04 \pm 1.22$ & $2.05 \pm 0.46$ & $2.15 \pm 1.05$ & $2.20 \pm 0.44$ & $2.08 \pm 0.58$ \\
& $(V-I)$ & $1.16 \pm 0.39$ & $1.14 \pm 1.17$ & $1.13 \pm 0.36$ & $1.13 \pm 0.99$ & $1.18 \pm 0.34$ & $1.17 \pm 0.45$ \\
& $(B-V)$ & $0.90 \pm 0.42$ & $0.90 \pm 1.21$ & $0.92 \pm 0.40$ & $1.02 \pm 1.06$ & $1.01 \pm 0.38$ & $0.91 \pm 0.49$ \\
Scatter $^{\mathrm{b}}{ }^{2}$ & $(B-I)$ & $0.067(0.032)$ & $0.083(0.048)$ & $0.076(0.076)$ & $0.099(0.074)$ & $0.102(0.073)$ & $0.084(0.084)$ \\
& $(V-I)$ & $0.064(0.037)$ & $0.038(0.022)$ & $0.054(0.054)$ & $0.072(0.070)$ & $0.066(0.046)$ & $0.042(0.042)$ \\
& $(B-V)$ & $0.038(0.040)$ & $0.061(0.050)$ & $0.034(0.034)$ & $0.084(0.062)$ & $0.063(0.057)$ & $0.048(0.048)$ \\
\hline
\end{tabular}

a Number of all early-type member galaxies (The value in parentheses means the number of early-type members after sigma clipping.).

${ }^{b}$ Scatter of all early-type member galaxies around the CMR (The value in parentheses means the CMR scatter.).

Table 4. Mean Colors, Ages, and Metallicities of the Early-Type Member Galaxies in Six Clusters

\begin{tabular}{cccccccccc}
\hline \hline Cluster & $\mathrm{N}$ & $\overline{(B-V)}$ & $\sigma_{B-V}{ }^{\mathrm{b}}$ & $\epsilon_{B-V}{ }^{\mathrm{c}}$ & $\overline{\left(I-K_{S}\right)^{\mathrm{a}}}$ & $\sigma_{I-K_{S}}{ }^{\mathrm{b}}$ & $\epsilon_{I-K_{S}}{ }^{\mathrm{C}}$ & Age $[$ Gyr] & Metallicity $(\mathrm{Z})$ \\
\hline $\mathrm{A} 1436$ & 10 & $0.921 \pm 0.012$ & 0.035 & 0.046 & $2.100 \pm 0.036$ & 0.109 & 0.102 & $3.1_{-0.2}^{+0.3}$ & $0.045_{-0.005}^{+0.004}$ \\
$\mathrm{~A} 1773$ & 14 & $0.979 \pm 0.018$ & 0.063 & 0.089 & $2.129 \pm 0.038$ & 0.144 & 0.146 & $5.2_{-0.9}^{+1.8}$ & $0.042_{-0.005}^{+0.006}$ \\
$\mathrm{~A} 1809$ & 13 & $0.954 \pm 0.012$ & 0.032 & 0.048 & $2.023 \pm 0.043$ & 0.145 & 0.117 & $6.1_{-1.1}^{+2.0}$ & $0.032_{-0.007}^{+0.006}$ \\
$\mathrm{~A} 2048$ & 13 & $1.075 \pm 0.018$ & 0.065 & 0.091 & $2.143 \pm 0.052$ & 0.178 & 0.146 & $19.6_{-6.2}^{+0.4}$ & $0.031_{-0.010}^{+0.009}$ \\
$\mathrm{~A} 2142$ & 31 & $1.032 \pm 0.010$ & 0.053 & 0.050 & $2.117 \pm 0.021$ & 0.116 & 0.136 & $11.9_{-1.4}^{+1.6}$ & $0.033_{-0.003}^{+0.003}$ \\
$\mathrm{~A} 2152$ & 11 & $0.936 \pm 0.025$ & 0.059 & 0.044 & $2.011 \pm 0.050$ & 0.122 & 0.079 & $5.1_{-1.6}^{+2.6}$ & $0.033_{-0.006}^{+0.006}$ \\
\hline
\end{tabular}

$a$ Mean color and its uncertainty estimated through random sampling test.

${ }^{b}$ Standard deviation of the colors.

$c$ Mean error in the colors.

\section{(b) Color-Magnitude Relations}

Figure 2 displays the $V-(B-I)$ color-magnitude diagrams of all the measured galaxies in each cluster, and Figure 3 displays the $M_{V}-(B-I)_{0}$ colormagnitude diagrams. In Figure 3 we plotted only the confirmed members (large symbols) and uncertain members (small symbols). We chose the $(B-I)$ color because it covers the widest range of wavelength in our optical bands.

A few features are noted in the color-magnitude diagrams. First, most early-type galaxies in each cluster show a linear sequence for the CMR: the brighter galaxies are the redder. Second, the CMR is tight in some clusters like A1436, and is less tight in other clusters like A2048 and A2142, considering the scatters of early-type member galaxies around the CMRs which are listed in Table 3 . Third, there is a secondary branch at a much redder color, $(B-I) \sim 2.8$, in the observed color-magnitude diagram of A2152. Most galaxies on the secondary branch are probably the members of the background cluster mentioned above. We consider the galaxies on the blue side of the dashed line in the $M_{V}$ $(B-I)_{0}$ color-magnitude diagram of A2152 to belong to A2152.
We carried out linear fitting to the data for earlytype member galaxies in each cluster after the application of sigma clipping algorithm. The results of fitting the CMR are summarized in Table 3, and are shown by straight lines in Figure 3 . The slopes for $M_{V}-(B-I)_{0}$ CMRs range from $-0.045 \pm 0.021$ (A2142) to $-0.096 \pm 0.058$ (A1773), and the intercepts ${ }^{\dagger}$ range from $2.04 \pm 1.22$ (A1773) to 2.20 \pm 0.44 (A2142). Thus the slopes and intercepts do not show any significant variation among the clusters. If we use the entire red-sequence galaxies for fitting, the ranges of the linear fit parameters become much narrower. The slope and intercept of A1773 become $-0.063 \pm 0.021$ and $2.09 \pm 0.43$, and those of A2142 become $-0.056 \pm 0.014$ and $2.16 \pm 0.30$.

\section{(c) Colors, Ages, and Metallicities}

Integrated colors of a stellar system are determined by its age and metallicity, and various combinations of optical and NIR colors have been used for estimating both the age and metallicity of a stellar system: $(U-R)$ and $(R-K)$ by Peletier \& Balcells (1996),

\footnotetext{
$\dagger$ Intercepts were calculated for $M_{V}=-20 \mathrm{mag}$
} 


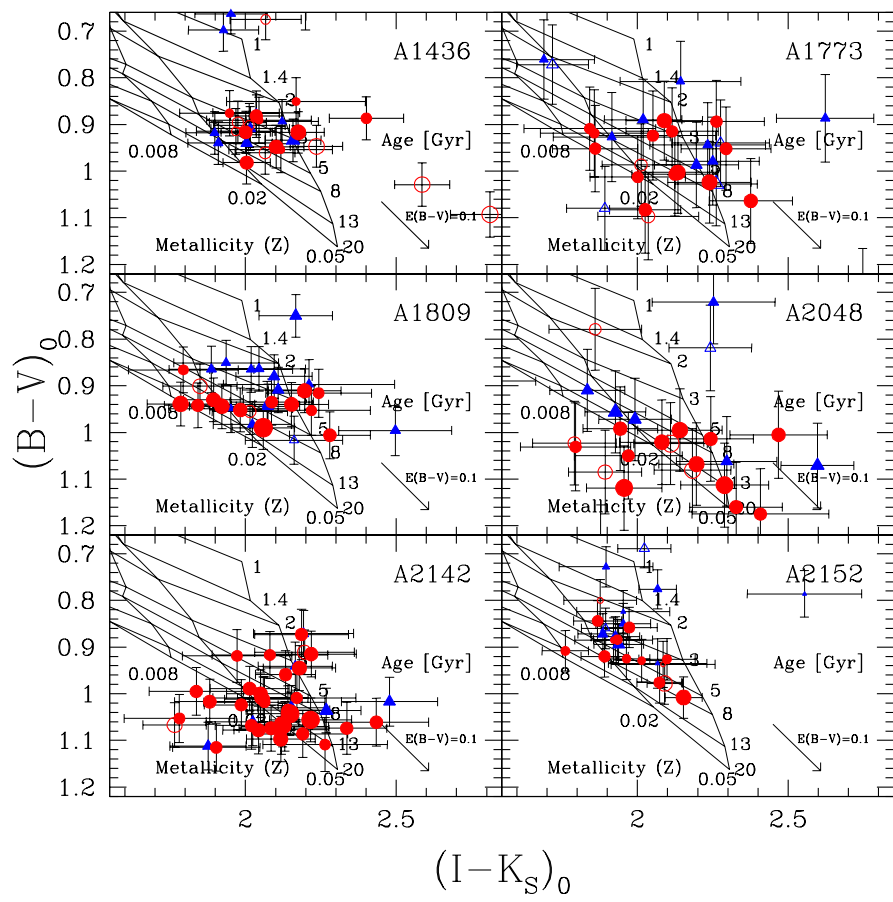

Fig. $\quad 4-\left(I-K_{S}\right)_{0}$ versus $(B-V)_{0}$ color-color diagrams of the galaxies in six clusters. The solid lines represent the BC03 SSP model grids for metallicity $(Z)$ and age. The symbols with color error bars represent BOAO-2MASS matched galaxies (circles : early-types, and triangles : latetypes). Filled and open symbols represent the confirmed members and uncertain members, respectively. The symbol size varies according to the $K_{S}$ luminosity : bigger for the more luminous galaxies. The vectors show the effect on the galaxy colors of increasing the extinction.

$(B-R)$ and $(R-K)$ by Bell \& de Jong $(2000),(V-I)$ and $(V-K)$ by Puzia et al. $(2002),(B-K)$ and $(J-K)$ by James et al. $(2006),(B-V)$ and $(B-K)$ by Li, Han, \& Zhang (2007), and $(B-V)$ and $(V-K)$ by H. Lee et al. (2007). Recently Li \& Han (2008) studied the ability of breaking the age-metallicity degeneracy using the stellar population synthesis model of Bruzual \& Charlot (2003) (called BC03 hereafter) for various combinations of the Johnson-Cousins-Glass $B V R I J H K$ system and the AB ugriz system. In their results all the best color pairs are combinations of the two systems, and the $(B-V)-(I-K)$ is the only pair composed of the Johnson-Cousins-Glass system. Therefore we decided to use these colors in this study.

Among several population synthesis models commonly in use today (Fioc \& Rocca-Volmerange 1997; BC03; Maraston 2005; Vázques \& Leitherer 2005; Schiavon 2007), we chose the BC03 model to estimate the ages and metallicities of galaxies. Although different initial mass functions (IMFs), chemical compositions, stellar evolutionary tracks, and spectral libraries are applied in the models, they return a fairly good agree-

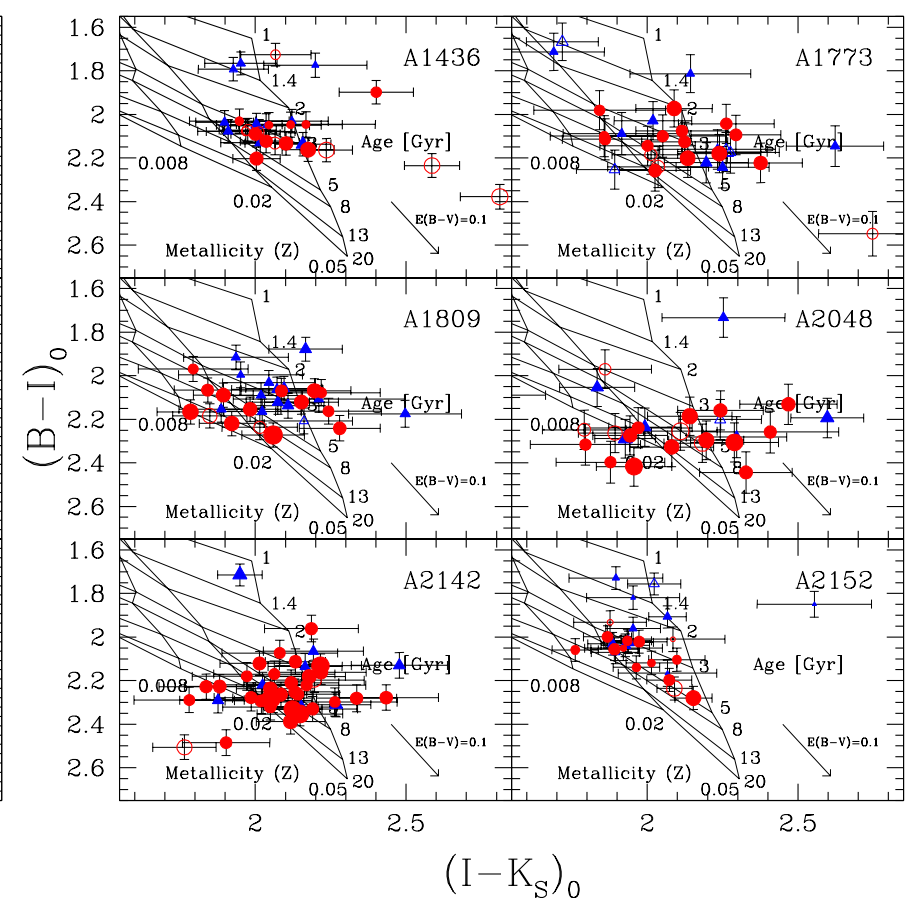

Fig. $5-\left(I-K_{S}\right)_{0}$ versus $(B-I)_{0}$ color-color diagrams of the galaxies in six clusters. The lines, symbols and vectors are the same as those in Fig. 4.

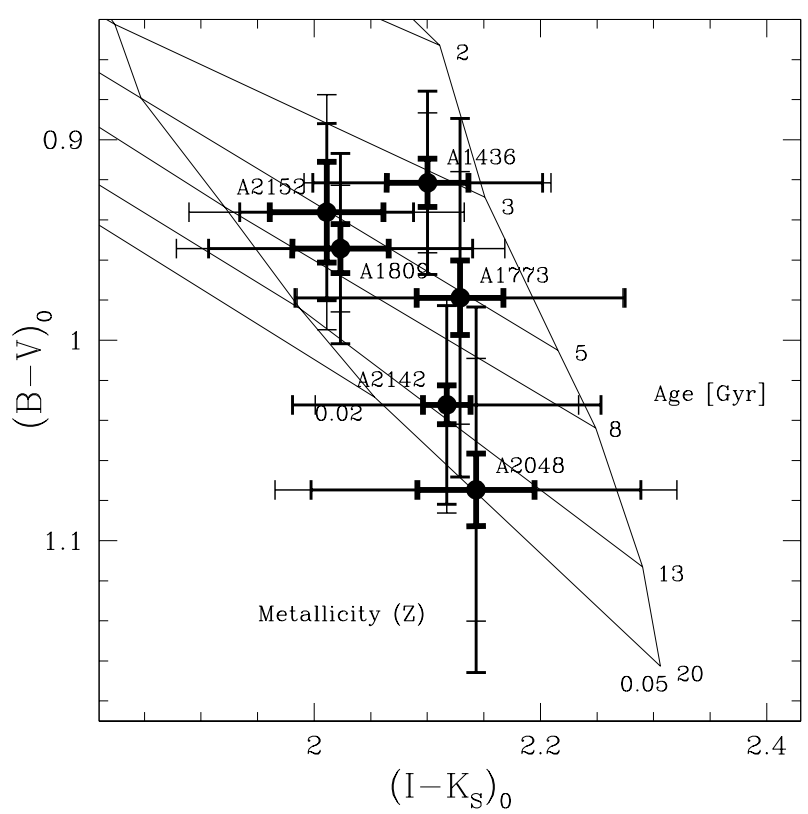

Fig. 6 - Mean colors of the early-type member galaxies in each cluster shown in $\left(I-K_{S}\right)_{0}$ versus $(B-V)_{0}$ colorcolor diagram. Thin, medium, and thick bars represent, respectively, the standard deviation of the colors, the mean error in the colors, and the uncertainty in the mean color. 


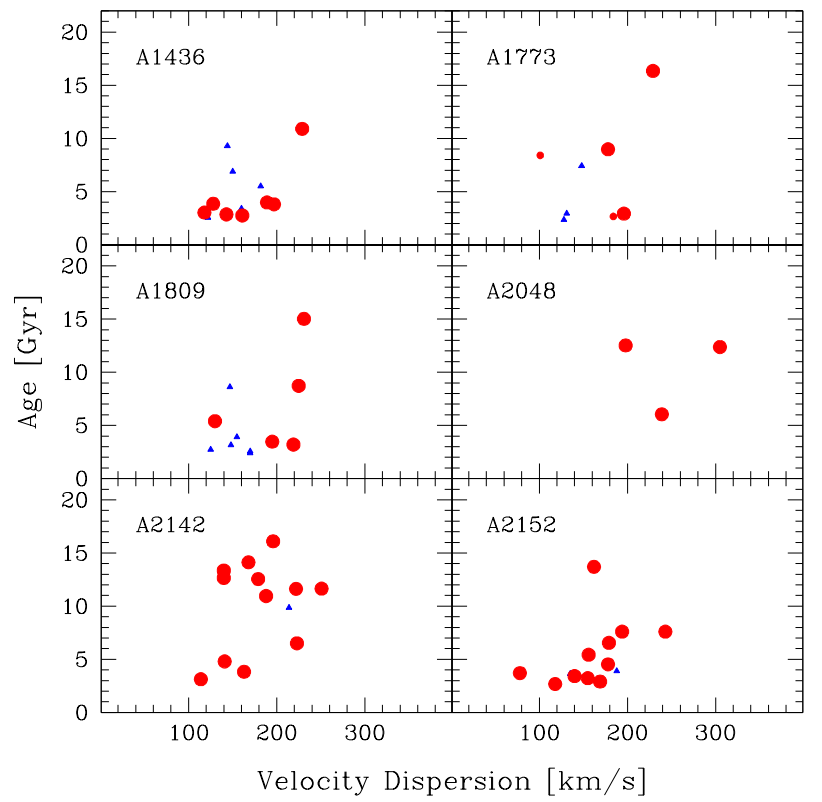

Fig. 7 - Age versus velocity dispersion diagrams of six clusters. Each symbol represents a member galaxy lying inside the SSP model grids (circles : early-types, and triangles : late-types). Large circles represent the red-sequence early-type galaxies.

ment for normal elliptical galaxies. James et al. (2006) described the effects of various factors (e.g., IMF slope, contribution of blue horizontal branch, mass loss in asymptotic giant branch, and $\alpha$-enhanced metal distribution) on the model prediction. If we infer the age for the $\alpha$-enhanced population using the scaled solar metal distribution model like BC03 model, we obtain a correct value for the old population ( $\sim 10 \mathrm{Gyr})$. However, there will be an overestimate of about 1 2 Gyr for the young population ( $\sim 3 \mathrm{Gyr})$. We adopted the Padova (1994) evolutionary tracks (Fagotto et al. 1994 and references therein) and the Salpeter IMF with a power law slope of -2.35 for the mass range of $0.1 M_{\odot}$ $\sim 100 M_{\odot}$, and assumed a simple stellar population (SSP; where all stars were instantaneously formed with the same chemical composition). This model covers ages from $10^{5}$ years to $2 \times 10^{10}$ years and metallicities from $\mathrm{Z}=0.0001$ to $\mathrm{Z}=0.05$.

The ages derived from the best fit with the SSP model do not represent the formation epoch or the mean age of the population, but represent the luminosityweighted ages (see Renzini 2006). In general, the luminosity-weighted age is sensitive to the youngest component of the population, so the age presented in this study should be regarded as the epoch of the last major star formation (i.e., lower age limit). Although we presented the estimated ages and metallicities of early-type and late-type galaxies in this study, the estimates of late-types are uncertain because the late-types are much more complicated systems having complex

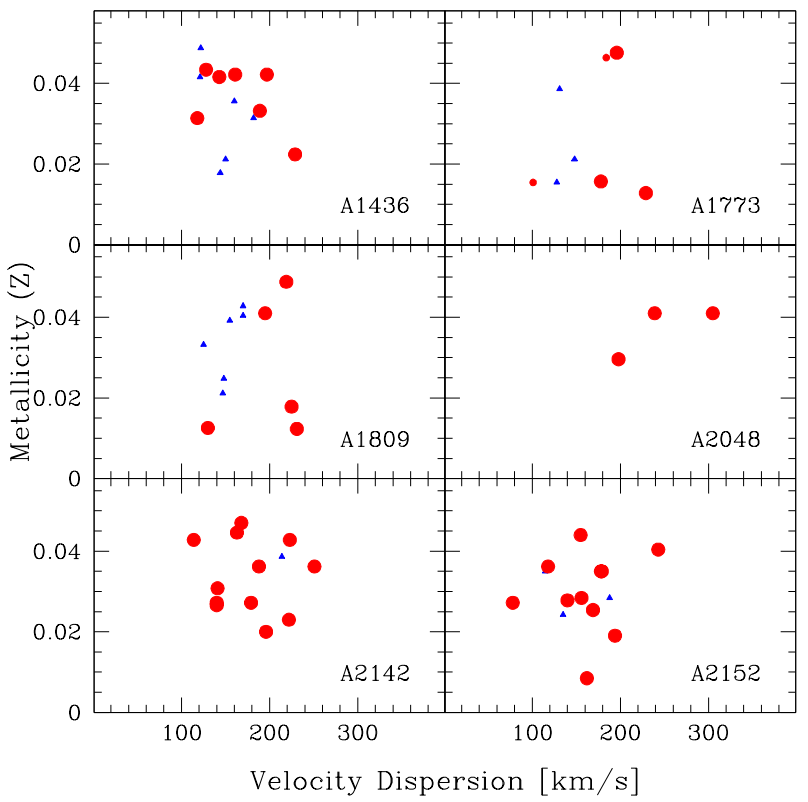

Fig. 8 - Metallicity versus velocity dispersion diagrams of six clusters. The symbols are the same as those in Fig. 7 .

star formation history (H. Lee et al. 2007). Moreover we did not correct the internal extinction of galaxies, so the colors of late-types used for fitting might be redder than intrinsic colors yielding higher metallicity or older age.

Figures 4 and 5 show the $\left(I-K_{S}\right)_{0}$ versus $(B-V)_{0}$ and $\left(I-K_{S}\right)_{0}$ versus $(B-I)_{0}$ color-color diagrams of the galaxies in six clusters with the BC03 SSP model grids, respectively. Most galaxies in each cluster are located within the boundary of the model grids, especially in A2152. However the number of outliers tends to increase with increasing redshifts and photometric errors. In A1436 and A2152, the ages of early-type galaxies are in a relatively narrow range, and the number of early-types older than $8 \mathrm{Gyr}$ are very small. We included $\left(I-K_{S}\right)_{0}$ versus $(B-I)_{0}$ color-color diagram to show this better. $(B-I)_{0}$ has a larger wavelength coverage and can be more sensitve to age than $(B-V)_{0}$.

Figure 6 displays the luminosity-weighted mean $(B-$ $V)_{0}$ and $\left(I-K_{S}\right)_{0}$ colors of early-type member galaxies in each cluster, which are also listed in Table 4. All clusters seem to have large color dispersions in Figures 4 and 5 , but the intrinsic dispersion ${ }^{\ddagger}$ should be much smaller because the color dispersion is mostly accounted for by color errors as shown in Figure 6. The mean $(B-V)_{0}$ colors range from 0.921 (A1436) to 1.075 (A2048), and the mean $\left(I-K_{S}\right)_{0}$ colors range from 2.011 (A2152) to 2.143 (A2048). The mean $(B-V)_{0}$ colors of six clusters are divided into two groups. One

$\ddagger \sigma_{\text {observed }}^{2}=\sigma_{\text {intrinsic }}^{2}+\sigma_{\text {error }}^{2}$ 
is 0.95 (A1436, A1773, A1809, and A2152), and the other is 1.05 (A2048 and A2142). On the other hand, the mean $\left(I-K_{S}\right)_{0}$ colors are similar to each other. We calculated the mean age and metallicity of early-type member galaxies in each cluster using the mean colors by interpolating the model grids. The mean ages range from 3.1 Gyr (A1436) to 19.6 Gyr (A2048), and the mean metallicities range from 0.031 (A2048) to 0.045 (A1436). The age dispersion among all six clusters ( $~ 5.4$ Gyr) is significantly larger than the mean error in ages $(\sim 2.4 \mathrm{Gyr})$. However the mean ages for the four clusters (A1436, A1773, A1809, and A2152), from 3.1 Gyr to 6.1 Gyr, show a much smaller dispersion, and are much smaller than the other two. The difference between the metallicity dispersion among six clusters $(\sim 0.004 \mathrm{Z})$ and the mean error in metallicities $(\sim 0.005 \mathrm{Z})$ is negligible. This seems to be in contrast to the result given by Jones, Smail, \& Couch (2000) and Smail et al. (2001) that the early-type galaxies in clusters have a large dispersion of metallicities rather than of ages. However it is difficult to directly compare between their result and ours because they did not present the mean age and metallicity of galaxies in individual clusters. It is needed to use a lager sample to check our finding.

\section{DISCUSSION}

It is widely known that more massive galaxies are older and more metal-rich than less massive galaxies from many studies based on a large sample of galaxies (Bernardi et al. 2005; Gallazzi et al. 2005, 2006; Nelan et al. 2005; Jimenez et al. 2007). In Figures 7 and 8, we display age and metallicity versus galaxy velocity dispersion, which is extracted from the SDSS catalog, for the member galaxies lying inside the SSP model grids. It is found in Figure 7 that age and velocity dispersion show a good correlation in Abell 2152, while it is not clear in other clusters. Figure 8 shows little correlation between metallicity and velocity dispersion in all six clusters. There are a few possibilities to explain these results. First, although there are in general good correlations between age and mass and between metallicity and mass in early-type galaxies, there are large dispersions as well. This dispersion may be due to diverse star formation history of the galaxies in clusters. Second, the number of galaxies in each cluster and the number of clusters in this study are too small to detect the correlations. Third, the correlations could disappear by large uncertainties in estimates of age and metallicity for individual galaxy.

Looking at Tables 1 and 4, the mean colors of early-type galaxies in each cluster tend to be redder with increasing redshift, especially in case of $(B-V)$ color. This result is not changed when we calculate the mean colors using only the early-type members brighter than $M_{K s}=-23.9$, faint end of early-type members in A2048. The differences in mean colors among several K-correction methods (i.e., Poggianti 1997; Bolzonella,
Miralles, \& Pelló 2000; Blanton et al. 2003) are smaller than $0.02 \mathrm{mag}$. Moreover the differences do not depend on the redshift, at least, up to $z \sim 0.1$. Thus the trend in mean colors should not be explained by these influences. On the other hand, it is also shown that the mean age is correlated with cluster velocity dispersion except for A2152. It is noted that the velocity dispersion of A2152 is problematic (Struble \& Rood 1999). This is in agreement with the recent finding reported by Rakos, Schombert, \& Odell (2008), and implies that galaxy formation in more massive cluster begins at earlier epoch.

\section{SUMMARY}

We presented $B V I$ photometry of galaxies in six nearby Abell galaxy clusters (A1436, A1773, A1809, A2048, A2142, and A2152) using the CCD imaging data obtained at the BOAO $1.8 \mathrm{~m}$ telescope. We combined these optical $B V I$ data with 2MASS NIR data, and used the spectroscopic redshifts and velocity dispersions from the SDSS. We analyzed the optical CMRs of early-type galaxies in each cluster, and estimated the ages and metallicities of bright galaxies using their $(B-V)$ and $\left(I-K_{S}\right)$ colors compared with the SSP model grids.

The six clusters show fairly tight CMRs, and there are no significant differences in the slopes or intercepts of the CMRs. In the age versus velocity dispersion and metallicity versus velocity dispersion diagrams, it is hard to see any strong correlations between them. Therefore it is difficult to tell whether the CMR is mainly driven by the age-mass relation or by the metallicity-mass relation. Among the clusters, the intrinsic dispersion of mean metallicities is small, while that of mean ages is relatively large. It is found that the early-type galaxies in more massive cluster tend to be older.

\section{ACKNOWLEDGEMENTS}

The authors thank N. Hwang for useful comments and acknowledge the support of the Brain Korea 21 program. The authors are grateful to the referee $\mathrm{T}$. Goto whose comments helped much to improve the original manuscript. This is supported in part by a grant (R01-2004-000-10490-0) from the Basic Research Program of the Korea Science and Engineering Foundation.

\section{REFERENCES}

Aaronson, M., 1978, The morphological distribution of bright galaxies in the UVK color plane,ApJ, 221, 103

Abell, G. O., 1958, The Distribution of Rich Clusters of Galaxies, ApJS, 3, 211

Abell, G. O., Corwin, H. G., \& Jr, Olowin, R. P., 1989, A catalog of rich clusters of galaxies, ApJS, 70, 1 
Adelman-McCarthy, J. K., et al., 2008, The Sixth Data Release of the Sloan Digital Sky Survey, ApJS, 175, 297

Bautz, L. P. \& Morgan, W. W., 1970, On the Classification of the Forms of Clusters of Galaxies, ApJ, 162,149

Bell, E. F. \& de Jong, R. S., 2000, The stellar populations of spiral galaxies, MNRAS, 312, 497

Bernardi, M., Sheth, R. K., Nichol, R. C., Schneider, D. P., \& Brinkmann, J., 2005, Colors, Magnitudes, and Velocity Dispersions in Early-Type Galaxies: Implications for Galaxy Ages and Metallicities, AJ, 129, 61

Bertin, E. \& Arnouts, S., 1996, SExtractor: Software for source extraction, A\&AS, 117, 393

Bessell, M. S., 1990, UBVRI passbands, PASP, 102, 1181

Blakeslee, J. P., Metzger, M. R., Kuntschner, H., \& Côté, P., 2001, Lensing in the Hercules Supercluster, AJ, 121,1

Blanton, M. R., Brinkmann, J., Csabai, I., Doi, M., Eisenstein, D., Fukugita, M., Gunn, J. E.,

Hogg, D. W., \& Schlegel, D. J., 2003, Estimating Fixed-Frame Galaxy Magnitudes in the Sloan Digital Sky Survey, AJ, 125, 2348

Bolzonella, M., Miralles, J. M., \& Pelló, R., 2000, Photometric redshifts based on standard SED fitting procedures, A\&A, 363, 476

Bower, R. G., Lucey, J. R., \& Ellis, R. S., 1992, Precision Photometry of Early Type Galaxies in the Coma and Virgo Clusters - a Test of the Universality of the Colour / Magnitude Relation - Part Two - Analysis, MNRAS, 254, 601

Bruzual, G. \& Charlot, S., 2003, Stellar population synthesis at the resolution of 2003, MNRAS, 344, 1000

Cox, A. N., 2000, Allen's Astrophysical Quantities, 4th ed., Springer-Verlag, New York

Fagotto, F., Bressan, A., Bertelli, G., \& Chiosi, C., 1994, Evolutionary sequences of stellar models with new radiative opacities. IV. $\mathrm{Z}=0.004$ and $\mathrm{Z}=0.008$, A\&AS, 105, 29

Ferreras, I., Charlot, S., \& Silk, J., 1999, TThe Age and Metallicity Range of Early-Type Galaxies in Clusters, ApJ, 521, 81

Fioc, M. \& Rocca-Volmerange B., 1997, PEGASE: a UV to NIR spectral evolution model of galaxies. Application to the calibration of bright galaxy counts, A\&A, 326, 950

Gallazzi, A., Charlot, S., Brinchmann, J., White, S. D. M., \& Tremonti, C. A., 2005, The ages and metallicities of galaxies in the local universe, MNRAS, 362, 41
Gallazzi, A., Charlot, S., Brinchmann, J., \& White, S. D. M., 2006, Ages and metallicities of early-type galaxies in the Sloan Digital Sky Survey: new insight into the physical origin of the colour-magnitude and the Mg2- $\sigma$ V relations, MNRAS, 370, 1106

Hogg, D. W., Blanton, M. R., Brinchmann, J., Eisenstein, D. J., Schlegel, D. J., Gunn, J. E., McKay, T. A., Rix, H., Bahcall, N. A., Brinkmann, J., \& Meiksin, A., 2004, The Dependence on Environment of the Color-Magnitude Relation of Galaxies, ApJ, 601,29

Holden, B. P., Stanford, S. A., Eisenhardt, P., \& Dickinson, M., 2004, Evolution in the Color-Magnitude Relation of Early-Type Galaxies in Clusters of Galaxies at $\mathrm{z}=1$, AJ, 127, 2484

James, P. A., Salaris, M., Davies, J. I., Phillipps, S., \& Cassisi, S., 2006, Optical/near-infrared colours of early-type galaxies and constraints on their star formation histories, MNRAS, 367, 339

Jarrett, T. H., Chester, T., Cutri, R., Schneider, S., Skrutskie, M., \& Huchra, J. P., 2000, 2MASS Extended Source Catalog: Overview and Algorithms, AJ, 119, 2498

Jimenez, R., Bernardi, M., Haiman, Z., Panter, B., \& Heavens, A. F., 2007, The Ages, Metallicities, and Star Formation Histories of Early-Type Galaxies in the SDSS, ApJ, 669, 947

Jones, L. A., Smail, I., \& Couch, W. J., 2000, Constraints on the Evolution of S0 Galaxies in Rich Clusters at Moderate Redshift, ApJ, 528, 118

Kauffmann, G. \& Charlot, S., 1998, Chemical enrichment and the origin of the colour-magnitude relation of elliptical galaxies in a hierarchical merger model, MNRAS, 294, 705

Kleinmann, S. G., Lysaght, M. G., Pughe, W. L., Schneider, S. E., Skrutskie, M. F., Weinberg, M. D., Price, S. D., Matthews, K., Soifer, B. T., \& Huchra, J. P., 1994, The Two Micron All Sky Survey, AP\&SS, 217, 11

Kodama, T., Arimoto, N., Barger, A. J., \& AragónSalamance, A., 1998, Evolution of the colourmagnitude relation of early-type galaxies in distant clusters, A\&A, 334, 99

Kodama, T., Tanaka, I., Kajisawa, M., Kurk, J., Venemans, B., De Breuck, C., Vernet, J., \& Lidman, C., 2007, The first appearance of the red sequence of galaxies in proto-clusters at $2<\sim \mathrm{z}<\sim 3$, MNRAS, 377,1717

Kron, R. G., 1980, Photometry of a complete sample of faint galaxies, ApJS, 43, 305

Landolt, A. U., 1992, UBVRI photometric standard stars in the magnitude range 11.5-16.0 around the celestial equator, AJ, 104, 340

Lee, M. G., 2003, On the Formation of Giant Elliptical Galaxies and Globular Clusters, JKAS, 36, 189 
Lee, H. C., Worthey, G., Trager, S. C., \& Faber, S. M., 2007, On the Age and Metallicity Estimation of Spiral Galaxies Using Optical and Near-Infrared Photometry, ApJ, 664, 215

Li, Z., Han, Z., \& Zhang, F., 2007, Potential of colors for determining age and metallicity of stellar populations, A\&A, 464, 853

Li, Z. \& Han, Z., 2008, Colour pairs for constraining the age and metallicity of stellar populations, MNRAS, 385,1270

Maraston, C., 2005, Evolutionary population synthesis: models, analysis of the ingredients and application to high-z galaxies, MNRAS, 362, 799

Markevitch, M. et al., 2000, Chandra Observation of Abell 2142: Survival of Dense Subcluster Cores in a Merger, ApJ, 541, 542

Nelan, J. E., Smith, R. J., Hudson, M. J., Wegner, G. A., Lucey, J. R., Moore, S. A. W., Quinney, S. J., \& Sunzeff, N. B., 2005, NOAO Fundamental Plane Survey. II. Age and Metallicity along the Red Sequence from Line-Strength Data, ApJ, 632, 137

Peletier, R. F. \& Balcells, M., 1996, Ages of Galaxies Bulges and Disks From Optical and Near-Infrared Colors, AJ, 111, 2238

Poggianti, B. M., 1997, K and evolutionary corrections from UV to IR, A\&AS, 122, 399

Puzia, T. H., Zepf, S. E., Kissler-Patig, M., Hilker, M., Minniti, D., \& Goudfrooij, P., 2002, Extragalactic globular clusters in the near-infrared. II. The globular clusters systems of NGC 3115 and NGC 4365, A\&A, 391,453

Rakos, K., Schombert, J., \& Odell, A., 2008, The Age of Cluster Galaxies from Continuum Colors, ApJ, 677, 1019

Renzini, A., 2006, Stellar Population Diagnostics of Elliptical Galaxy Formation, ARA\&A, 44, 141

Sandage, A. \& Visvanathan, N., 1978, The colorabsolute magnitude relation for $\mathrm{E}$ and $\mathrm{S} 0$ galaxies. II - New colors, magnitudes, and types for 405 galaxies, ApJ, 223, 707

Schiavon, R. P., 2007, Population Synthesis in the Blue. IV. Accurate Model Predictions for Lick Indices and UBV Colors in Single Stellar Populations, ApJS, 171,146

Schlegel, D. J., Finkbeiner, D. P., \& Davis, M., 1998, Maps of Dust Infrared Emission for Use in Estimation of Reddening and Cosmic Microwave Background Radiation Foregrounds, ApJ, 500, 525

Smail, I., Kuntschner, H., Kodama, T., Smith, G. P., Packham, C., Fruchter, A. S., \& Hook, R. N., 2001, A photometric study of the ages and metallicities of early-type galaxies in A 2218, MNRAS, 323, 839

Smith, R. J., Hudson, M. J., Nelan, J. E., Moore, S. A. W., Quinney, S. J., Wegner, G. A., Lucey, J. R.,
Davies, R. L., Malecki, J. J., Schade, D., \& Suntzeff, N. B., 2004, NOAO Fundamental Plane Survey. I. Survey Design, Redshifts, and Velocity Dispersion Data, AJ, 128, 1558

Smith, R. J., Hudson, M. J., Lucey, J. R., Nelan, J. E., \& Wegner, G. A., 2006, The NOAO Fundamental Plane Survey - III. Variations in the stellar populations of red-sequence galaxies from the cluster core to the virial radius, MNRAS, 369, 1419

Stanford, S. A., Eisenhardt, P. R., \& Dickinson, M., 1998, The Evolution of Early-Type Galaxies in Distant Clusters, ApJ, 492, 461

Terlevich, A. I., Caldwell, N., \& Bower, R. G., 2001, The colour-magnitude relation for galaxies in the Coma cluster, MNRAS, 326, 1547

Vázques, G. A. \& Leitherer, C., 2005, Optimization of Starburst99 for Intermediate-Age and Old Stellar Populations, ApJ, 621, 695,

Worthey, G.,1994, Comprehensive stellar population models and the disentanglement of age and metallicity effects, ApJS, 95, 107

Yoon, J. H., Schawinski, K., Sheen, Y.-K., Ree, C. H., \& Yi, S. K., 2008, A Spectrophotometric Search for Galaxy Clusters in SDSS, ApJS, 176, 414

York, D. G., et al., 2000, The Sloan Digital Sky Survey: Technical Summary, AJ, 120, 1579 